\title{
Endoscopic ultrasound is inadequate to determine which T1/T2 esophageal tumors are candidates for endoluminal therapies
}

\author{
Edward J. Bergeron, MD, Jules Lin, MD, Andrew C. Chang, MD, Mark B. Orringer, MD, and \\ Rishindra M. Reddy, MD
}

\begin{abstract}
Objectives: Esophageal endoscopic ultrasound is now regarded as essential in the staging of esophageal carcinoma. There is an increasing trend toward endoluminal therapies (ie, endoscopic mucosal resection and radiofrequency ablation) for pre-cancer or early-stage cancers because of concerns of high morbidity associated with esophagectomy. This study reviews our institutional experience with preoperative endoscopic ultrasound staging of early esophageal cancers in patients who underwent an esophagectomy to evaluate the accuracy of staging by endoscopic ultrasound and how this affects treatment recommendations.
\end{abstract}

\begin{abstract}
Methods: A prospective esophagectomy database of all patients undergoing an esophagectomy for esophageal cancer at a single high-volume institution was retrospectively reviewed for patients with early-stage esophageal cancer. This study analyzed patients with clinical Tis to $\mathrm{T} 1$ disease, as predicted by preoperative endoscopic ultrasound, and correlated this with the pathologic stages after esophagectomy. The surgical outcomes were evaluated to assess the safety of esophagectomy as a treatment modality.
\end{abstract}

Results: From 2005 to 2011, 107 patients (93 male, 14 female) with a mean age of 66 years (range, 39-91 years) were staged by preoperative endoscopic ultrasound to have esophageal high-grade dysplasia, carcinoma in situ, or T1 cancer and underwent an esophagectomy. Tumor depth was correctly staged by endoscopic ultrasound in only $39 \%$ (23/59) of pT1a tumors (invading into the lamina propria or muscularis mucosa) and $51 \%(18 / 35)$ of pT1b tumors (submucosal). Of the endoscopic ultrasound-staged cT1a-lpN0 lesions, there were positive lymph nodes in $15 \%$ of pathologic specimens (2/13). Patients with pT1a-mm lesions had a $9 \%$ rate of pathologic lymph node involvement (1/11), and those with pT1b tumors had a $17 \%$ rate of lymph node spread (6/35). Esophagectomy was performed in all 107 patients with a 30-day mortality rate of less than $1 \%(1 / 107)$.

Conclusions: The sensitivity and specificity of endoscopic ultrasound for determining true pathologic staging are poor for early-stage esophageal cancers. Lesions thought to be cT1a-lpN0 by endoscopic ultrasound have at least $\mathrm{pN} 1$ disease in $15 \%$ of cases. Endoluminal therapy of these lesions based on endoscopic ultrasound undertreats a significant number of patients. Esophagectomy is still the standard therapy for early-stage esophageal cancers in the majority of patients. (J Thorac Cardiovasc Surg 2014;147:765-73)

The National Cancer Institute projects that for 2013 in the United States, there will be 17,990 new patient diagnoses of esophageal cancer and 15,210 deaths from the disease. ${ }^{1}$ The high mortality associated with esophageal cancer is attributed, at least partially, to the early metastasis and late clinical presentation of the disease. Although in historical series, patients with esophageal cancer presented in advanced stages, more recent series show that with increased surveillance endoscopy, many patients $(\sim 33 \%)$ are diagnosed with early-stage (T1) lesions. ${ }^{2}$ The surveillance of Barrett's esophagus (BE), a premalignant lesion

From the Section of Thoracic Surgery, University of Michigan, Ann Arbor, Mich Disclosures: Authors have nothing to disclose with regard to commercial support.

Read at the 92nd Annual Meeting of The American Association for Thoracic Surgery, San Francisco, California, April 28-May 2, 2012.

Received for publication April 25, 2012; revisions received Oct 1, 2013; accepted for publication Oct 11, 2013; available ahead of print Dec 5, 2013.

Address for reprints: Rishindra M. Reddy, MD, University of Michigan, 1500 E

Medical Center Drive, Ann Arbor, MI 48109 (E-mail: reddyrm@med.umich.edu). 0022-5223/ $\$ 36.00$

Copyright (c) 2014 by The American Association for Thoracic Surgery

http://dx.doi.org/10.1016/j.jtcvs.2013.10.003 with the potential to progress to esophageal adenocarcinoma (EAC), allows for potential curative intervention for lesions detected at early stages. The management of BE traditionally was based on acid suppression therapy and serial endoscopic surveillance with methodical biopsies. Esophagectomy has been the standard management of Tis and invasive EACs. Despite its success in the eradication of $\mathrm{BE}$ and esophageal cancers, esophagectomy is associated with a high morbidity and mortality. ${ }^{3}$ Newer endoscopicbased therapies have been proposed as alternatives to esophagectomy for the treatment of Tis or early invasive EAC. These endoscopic treatments involve the resection or destruction of the concerning esophageal mucosa. Endomucosal resection (EMR) is an endoscopic-based method of resecting esophageal mucosa, allowing for examination of tumor depth ( $\mathrm{T}$ stage). Radiofrequency ablation (RFA) and photodynamic therapy (PDT) are ablative endoscopic-based therapies that destroy the targeted tissue but do not allow for the pathologic assessment of the tumor. ${ }^{4}$ Despite the recent successes of EMR, RFA, and PDT in abolishing esophageal mucosal lesions, 


\section{Abbreviations and Acronyms \\ $\mathrm{BE}=$ Barrett's esophagus \\ EAC $=$ esophageal adenocarcinoma \\ $\mathrm{EMR}=$ endomucosal resection \\ EUS $=$ endoscopic ultrasound \\ $\mathrm{NPV}=$ negative predictive value \\ PDT $=$ photodynamic therapy \\ PET $=$ positron emission tomography \\ PPV $=$ positive predictive value \\ RFA $=$ radiofrequency ablation \\ THE $=$ transhiatal esophagectomy}

these therapies do not address the invasion of deeper layers or evaluate or treat potential nodal spread.

Endoscopic ultrasound (EUS) is a component of preoperative staging that directs both esophagectomy and endoscopic-based therapies. ${ }^{5}$ EUS allows for the evaluation of esophageal tumors and involved lymph nodes. It is the principal tool for determining the clinical stage of the esophageal cancer and the subsequent therapy, which may include endoscopic-based treatment or surgery, neoadjuvant chemoradiation followed by surgery, or definitive chemotherapy with or without radiation. The aims of this study were to correlate the final pathology report on esophagectomy specimens in patients diagnosed with cTis and early-stage esophageal cancers (cTis-cT1), as determined by EUS, and determine the utility of EUS in planning therapeutic endoluminal treatments for early-stage esophageal cancers. The study was designed to simulate the clinician's decision-making pathway with currently used EUS-based staging for early-stage esophageal cancers. The perioperative outcomes after esophagectomy also were reviewed to characterize the risks for patients with early-stage esophageal cancer at the University of Michigan.

\section{MATERIALS AND METHODS}

Data for 447 patients in a prospectively collected database who had an esophagectomy for Tis or esophageal cancer at a single, high-volume institution between July 7, 2005, and July 7, 2011, were retrospectively reviewed. The patients had early-stage, Tis or T1, esophageal cancer by clinical staging with biopsies. All patients underwent a preoperative EUS, and those diagnosed as having T2 or greater disease depth or biopsy-proven N1 were excluded. Patients with EUS suspected N1 disease (but not biopsy confirmed) who proceeded directly to esophagectomy were included. Suspected lymphadenopathy was defined by the following criteria: hypoechoic pattern, spherical contour, presence of a distinct border, and short-axis diameter of $6 \mathrm{~mm}$ or greater. ${ }^{6}$ Patients with lesions that extended to the cardia of the stomach or who received preoperative chemotherapy or radiotherapy also were excluded. In the remaining 107 patients, preoperative endoscopic examinations, pathology reports, operative intervention, cancer staging, and perioperative esophagectomy outcomes were reviewed.

Standard guidelines for esophageal cancer staging based on the 7th edition of the American Joint Committee on Cancer $^{7}$ were used.
This study analyzed the substages of Tis and T1 disease as predicted by preoperative EUS and the correlation with pathologic $\mathrm{T}$ and $\mathrm{N}$ stages.

The preoperative EUS and pathologic T staging was defined as follows:

- Tis: tumor confined to the epithelium and not invading the lamina propria

- T1a-lp: tumor invading into but not through the lamina propria

- T1a-mm: tumor invasion into but not through muscularis mucosa

- T1b: tumor invasion into but not through the submucosa

If a tumor penetrated more than 1 of the classified tumor depths described, it was classified as the deepest involved depth. All EUS studies were performed by experienced endosonographers using Olympus 7.5 and $12 \mathrm{MHz}$ probes (Olympus, Center Valley, Pa). No EUS examinations were performed by thoracic surgeons. Higher frequency probes $(20$ and $30 \mathrm{MHz})$ were not routinely used, nor was their use specifically recorded. Regardless of the probe type used, a review of the endosonography report resulted in the clinical EUS stage used. Any EUS reading of possible metastatic disease within a lymph node was recorded as $\mathrm{cN} 1$ disease preoperatively. The $\mathrm{cN} 1$ nodes met the following sonographic criteria: size greater than $1 \mathrm{~cm}$, hypoechoic, distinct margins, and round shape. ${ }^{6}$ In general, cT or $\mathrm{cN}$ denotes clinical stage determined by EUS, and $\mathrm{pT}$ or $\mathrm{pN}$ denotes pathologic stage determined by histopathology.

All esophagectomies were performed by a group of 6 university-based general thoracic surgeons at a single institution. The accuracy of preoperative EUS staging was determined by comparing the most recent EUS staging with the surgical specimen final pathologic reports. Sensitivity, specificity, accuracy, positive predictive value (PPV), and negative predictive value (NPV) for metastatic lymph node involvement by EUS were calculated using standard formulas. The Fisher exact test was used to determine statistical significance between groups.

Perioperative esophagectomy outcomes were analyzed and included postoperative hospital length of stay, anastomotic leak, atrial fibrillation, hoarseness, chylothorax, deep venous thrombosis, wound infection, and perioperative mortality. Perioperative mortality was defined as death within 30 days of operation or in-hospital mortality. This study was approved by the University of Michigan Institutional Review Board.

\section{RESULTS}

From July 2005 to July 2011, 107 patients (93 male, $87 \%$ ) with a median age of 66 years (range, 39-91 years) were staged by preoperative EUS to have esophageal high-grade dysplasia, carcinoma in situ, or T1 esophageal cancer and underwent an esophagectomy. Table 1 summarizes the demographic and preoperative clinical information of the patients studied. The median timing from EUS to esophagectomy was 59 days; $98.1 \%$ (105) were Caucasian, and $90.7 \%$ (97) had distal esophageal tumors. The most common histology was adenocarcinoma of the esophagus $(89,83.1 \%)$, and $62.6 \%$ (67) had Barrett's metaplasia in the background of Tis or invasive cancer. Only 8 patients $(7.5 \%)$ had squamous cell cancer.

Table 2 displays the preoperative clinical EUS TNM staging (cT and $\mathrm{cN}$ ) and post-esophagectomy pathologic TNM staging ( $\mathrm{pT}$ and $\mathrm{pN}$ ) for all 107 patients. All patients were clinically and pathologically M0. All patients had Tis or invasive cancer on pathology (pTis or pT1). No patients after esophagectomy had an absence of carcinoma in situ or invasive disease on final post-esophagectomy pathology. None of the 107 patients studied had preoperative or pathologic distant metastatic disease $(\mathrm{cM}$ and $\mathrm{pM}=0)$. 
TABLE 1. Patient demographics and clinical characteristics

\begin{tabular}{lc}
\hline Study population, $\mathrm{n}$ & 107 \\
Age, $\mathrm{y}$, median (range) & $66(39-91)$ \\
Gender, $\mathrm{n}(\%)$ & \\
$\quad$ Male & $93(87 \%)$ \\
Female & $14(13 \%)$ \\
Race, Caucasian/non-Caucasian, $\mathrm{n}(\%)$ & \\
$\quad$ Caucasian & $105(98.1 \%)$ \\
$\quad$ Non-Caucasian & $2(1.9 \%)$ \\
Tumor location, $\mathrm{n}(\%)$ & \\
Proximal esophagus & $1(0.9 \%)$ \\
Mid esophagus & $9(8.4 \%)$ \\
Distal esophagus-GE junction & $97(90.7 \%)$ \\
Histopathology & \\
Any presence of Barrett's metaplasia, $\mathrm{n}(\%)$ & $67(62.6 \%)$ \\
Adenocarcinoma & $89(83.2 \%)$ \\
Squamous & $8(7.5 \%)$ \\
Carcinoma in situ & $9(8.4 \%)$ \\
Adenosquamous & $1(0.9 \%)$ \\
Type of surgical resection, $\mathrm{n}(\%)$ & \\
THE with CEGA & $105(98.2 \%)$ \\
3-hole esophagectomy & $2(1.8 \%)$ \\
\hline
\end{tabular}

CEGA, Cervical esophagogastric anastomosis; $G E$, gastroesophageal; THE, transhiatal esophagectomy.

\section{Tumor Evaluation}

The average tumor size in the esophagectomy specimens, for those with visible lesions, was $8.9 \mathrm{~mm}$ (median, $8.0 \mathrm{~mm}$ ). Nine patients had resection for Tis or carcinoma confined to the epithelium (pTis). The majority of the tumors, $55 \%$ (59), were confined to the mucosa (pT1alp + pT1a-mm), whereas $32.7 \%$ (35) had only submucosal (pT1b) deepest involvement. All esophagectomy specimens had pTis or invasive disease. Tumor depth was correctly staged by EUS in only 39\% (23/59) of pT1a tumors, those tumors invading into the lamina propria or muscularis mucosa, and 51\% (18/35) of tumors with submucosal involvement (pT1b). EUS understaged tumor depth in $32 \%(19 / 59)$ of pT1a tumors and $49 \%$ (17/35) of pT1b tumors. Overstaging of tumor depth by EUS occurred in $29 \%$ (17/59) pT1a tumors and 51\% (18/35) of pT1b tumors.

In particular, 3 tumors on EUS invaded into but not through the muscularis mucosa (T1a-muscularis mucosa);
2 of these tumors were staged as pT2 EACs, and 1 of these tumors was staged as a pT3 squamous cell carcinoma on histology.

The sensitivity of EUS for detecting cT1a lamina propria tumor invasion was $4.16 \%$ with a specificity of $81.35 \%$. Overall sensitivity was $72 \%$ for EUS-staged tumors with invasion superficial to the submucosa $(<\mathrm{cT} 1 \mathrm{~b})$ and specificity of $48.7 \%$.

\section{Lymph Node Evaluation}

A total of 1083 lymph nodes underwent pathologic analysis, and 14 lymph nodes in 9 patients contained metastatic disease. All 9 patients had adenocarcinomas, were understaged on tumor depth, and thought to have no lymph node involvement on preoperative EUS (cN0). Of these 9 patients, 1 had invasive disease on pathology to the level of the muscularis mucosa (pT1a-mm), 6 to the level of the submucosa (pT1b), and the remaining 2 patients had pT2 disease on final pathology (invasion to the muscularis propria). Thus, 8 of the 9 patients with positive pathologic lymph nodes had tumor invasion into the submucosal level or further. Furthermore, 2 of these 9 patients had 3 or more lymph nodes containing metastatic disease and were subsequently classified as having $\mathrm{pN} 2$ disease. One of these 2 patients with $\mathrm{pN} 2$ had tumor invasion into the submucosa (pT1b) and the other to the muscularis propria (pT2). Figure 1 shows the prevalence of metastatic lymph nodes $(\mathrm{pN})$ based on the pathologic tumor penetration depth. It is notable that 48 patients with pathologic T1a-lp disease had no evidence of lymph node spread. One of 11 patients with T1-mm disease did have lymph node involvement. Only 4 patients had pT2 or pT3 disease.

Of the EUS-staged cN0 lesions, there were pathologically positive lymph nodes in $15 \%(2 / 13)$ of cT1a lamina propria lesions and 18\% (5 of 28) of cT1a muscularis mucosa lesions. More specifically, 2 EUS-staged cT1a muscularis mucosal lesions had $\mathrm{pN} 2$ disease on final pathology, and again, both of these lesions were staged by EUS as cN0. These 2 tumors were EACs. Overall, EUS understaged $10 \%$ (9/90) of patients with lymph nodes

TABLE 2. cTNM versus pTNM for early-stage esophageal cancers

\begin{tabular}{|c|c|c|c|c|c|c|c|c|c|c|c|c|c|}
\hline & \multirow[b]{2}{*}{ cTNM } & \multicolumn{12}{|c|}{ pTNM } \\
\hline & & pT0 & pTis & pT1a-lp & pT1a-mm & pT1b (sm) & pT2 & pT3 & $T$ total & pNo & pN1 & pN2 & $\mathbf{N}$ total \\
\hline \multirow[t]{8}{*}{ All cNO } & cT0 & 0 & 1 & 12 & 3 & 1 & 0 & 0 & 17 & 17 & 0 & 0 & 17 \\
\hline & cTis & 0 & 2 & 2 & 2 & 0 & 0 & 0 & 6 & 6 & 0 & 0 & 6 \\
\hline & cT1a-lp & 0 & 2 & 2 & 1 & 8 & 0 & 0 & 13 & 11 & 2 & 0 & 13 \\
\hline & cT1a-mm N0 & 0 & 2 & 11 & 3 & 8 & 2 & 1 & 27 & 22 & 3 & 2 & 27 \\
\hline & cT1a-mm N1 & 0 & 0 & 6 & 0 & 0 & 0 & 0 & 6 & 6 & 0 & 0 & 6 \\
\hline & $\mathrm{cT} 1 \mathrm{~b}(\mathrm{sm}) \mathrm{N} 0$ & 0 & 1 & 11 & 2 & 12 & 1 & 0 & 27 & 25 & 2 & 0 & 27 \\
\hline & $\mathrm{cT} 1 \mathrm{~b}(\mathrm{sm}) \mathrm{N} 1$ & 0 & 1 & 4 & 0 & 6 & 0 & 0 & 11 & 11 & 0 & 0 & 11 \\
\hline & Total & 0 & 9 & 48 & 11 & 35 & 3 & 1 & 107 & 98 & 7 & 2 & 107 \\
\hline
\end{tabular}

Tla-lp, Furthest invasion into lamina propria; Tla-mm, furthest tumor invasion into muscularis muscosa; $T 1 b(s m)$, furthest invasion into submucosa. 


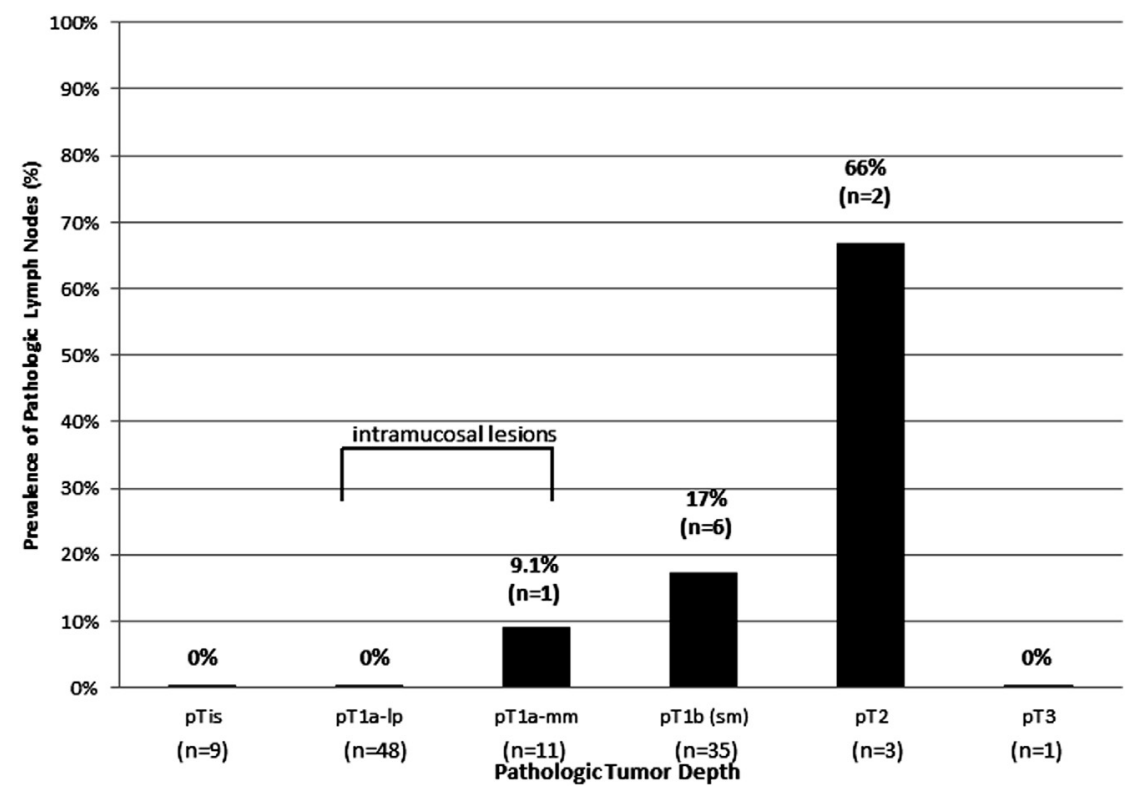

FIGURE 1. Prevalence of metastatic pathologic nodes based on pathologic tumor depth.

metastases preoperatively staged as cN0. Furthermore, 17 patients were thought to have EUS-staged nodes suspicious of metastatic disease (cN1), yet all were node negative after evaluation of the esophagectomy specimens. Figure 2 compares the prevalence of predicted lymph node involvement on EUS $(\mathrm{cN})$ and positive final pathologic examination $(\mathrm{pN})$ in tumors based on their clinical tumor depth staging (cT). There was no statistically significant difference between the groups, but 7 patients who were thought to have T1a tumors by EUS had positive lymph node involvement.

The sensitivity, specificity, PPV, NPV, and accuracy of EUS for lymph node involvement $(\mathrm{cN})$ were calculated. EUS sensitivity for lymph node involvement $(\mathrm{cN} 1)$ was $0 \%$ with a PPV of $0 \%$ because none of the patients with
EUS predicted to have lymph node involvement actually had pathologically positive lymph nodes. Conversely, the high specificity of $90 \%$ for EUS node involvement is likely due to the large number of patients (89/107) with pNO.

\section{Perioperative Characteristics}

A total of 105 of the 107 patients underwent a transhiatal esophagectomy (THE), and the remaining 2 patients had a 3-hole esophagectomy. The median intraoperative blood loss was $250 \mathrm{~mL}$ (range, $50-1400 \mathrm{~mL}$ ). A single patient required splenectomy at the time of esophagectomy because of intraoperative splenic injury. Seven patients received blood transfusion during their hospitalization.

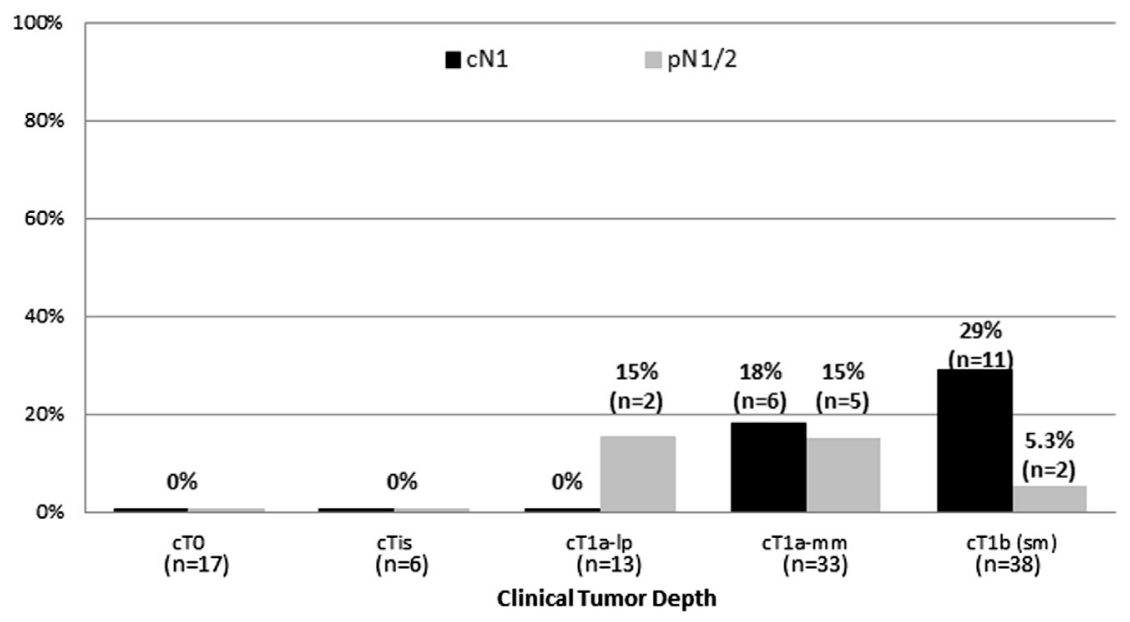

FIGURE 2. Prevalence of metastatic lymph nodes in EUS early-stage cT0-cT1 esophageal tumors: EUS cN versus esophagectomy pN. EUS, Endoscopic ultrasound. 
TABLE 3. Perioperative outcomes in patients undergoing esophagectomy for early-stage cT0-cT1 esophageal tumors

\begin{tabular}{lc}
\hline Hospital or 30-d mortality, n (\%) & $1(0.9 \%)$ \\
Blood loss, mL, median (range) & $250(50-1400)$ \\
Perioperative blood transfusion, patients, n (\%) & $7(6.5 \%)$ \\
Hospital length of stay, d, median (range) & $8(7-48)$ \\
Need for readmission, patients, n (\%) & $7(6.5 \%)$ \\
$\quad$ Days from discharge to readmission, median (range) & $3(0-16)$ \\
$\quad$ Readmission length of stay, d, median (range) & $4(2-12)$ \\
Need for reoperation, n (\%) & $4(3.7 \%)$ \\
Total complications, patients, n (\%) & $35(32.7 \%)$ \\
Complications (most common) & \\
Anastomotic leak & $12(11.2 \%)$ \\
Atrial fibrillation & $12(11.2 \%)$ \\
\hline
\end{tabular}

The median inpatient hospital stay was 8 days (range, 7-48 days). Table 3 summarizes the esophagectomy perioperative outcomes. There were no airway injuries or gastrointestinal obstructions. The 30-day postoperative mortality rate was less than $1 \%(1 / 107)$. The single death occurred in a patient who had an unexplained respiratory arrest on postoperative day 2 and for whom the family withdrew care. He died on postoperative day 4, and an autopsy was denied.

\section{DISCUSSION}

Carcinoma in situ (Tis) of the esophagus and early-stage esophageal cancers are currently undergoing alternative treatment algorithms at many institutions in place of esophagectomy. ${ }^{8-10}$ EUS has had a major role in the treatment planning of esophageal cancers, even for these alternative treatments. Endoluminal treatments currently consist primarily of EMR, RFA, and PDT. Incomplete endoluminal resection or ablation may occur in patients with tumor depth more advanced than demonstrated by EUS, and these patients would have had a complete resection and better staging with an esophagectomy. We analyzed the results of esophagectomy specimens for biopsy-proven Tis-T1 esophageal cancers on the basis of on their preoperative EUS staging. Our data show that esophagectomy is safe and effective in early-stage esophageal cancers with a mortality rate less than $1 \%$.

EMR produces a tissue specimen that allows for potential accurate pathologic staging of superficial lesions, but does not allow for accurate lymph node staging. Previous studies have shown EMR can be definitive treatment for superficial lesions extending to the muscularis mucosa. ${ }^{2}$ Large lesions can be removed piecemeal; however, there is an increased risk of metachronous neoplasia if a complete resection is not achieved. ${ }^{11}$ The risk of metachronous neoplasia can be limited with robust surveillance endoscopy and biopsy. ${ }^{9}$ The second popular alternative treatments to esophagectomy are based on destruction of the abnormal mucosa, allowing for regeneration with squamous epithelium. PDT and RFA have grown in popularity for the treatment of Tis and RFA for even intramucosal cancer if combined with EMR. ${ }^{9}$ Drawbacks for these methods are that there is no resulting pathologic specimen and lymph node status is not directly addressed.

EUS is an important component for clinical staging of the full spectrum of esophageal cancer. For Tis-T1 stage esophageal cancers, EUS is relied on for the staging of lymph nodes and traditionally for the depth of tumor invasion. If by EUS the lymph nodes appear to be free of metastatic disease, and tumor invasion is superficial, some have advocated for endoluminal therapy of the esophageal lesions. ${ }^{12}$ However, our data show that 9 patients $(8.4 \%)$ thought to be $\mathrm{cN} 0$ on EUS had pN1 on final pathology. If these patients were to undergo endoluminal therapy, they would have residual metastatic lymph node disease left behind and would not have received multimodal treatment with chemoradiotherapy until the disease had progressed. On final pathologic staging, 8 of the 9 patients who had lymph node metastases did have tumor penetration into or through the submucosa (Figure 1), because none of the 48 patients with pT1a-lp tumors had lymph node involvement, and only 1 patient with pT1a-mm had positive lymph nodes. In the T1b-sm group, $17 \%$ had lymph node metastases. This is consistent with other reports that submucosal tumors have $7.5 \%$ to $45 \%$ pathologically positive nodes with increasing tumor depth. ${ }^{2,13,14}$ Of greatest concern is that the majority (69.2\%) of our clinically staged T1a-lp tumors were understaged by $\mathrm{T}$ depth, and $15 \%$ had lymph node metastases (Figure 2). This group would have been undertreated on the basis of EUS staging. All patients studied had EUS performed with 7.5 and $12 \mathrm{MHz}$ probes. These probes are those that are used routinely by referring gastroenterologists. Higher-frequency 20 and $30 \mathrm{MHz}$ probes were not systematically used or evaluated. These specific probes are not typically used for nodal staging, but their routine incorporation into the EUS armamentarium may improve tumor depth clinical staging.

This study investigates all types of early-stage esophageal cancers (ie, adenocarcinoma and squamous cell carcinoma) in all locations of the esophagus. Those malignancies located in the cardia were excluded. Squamous cell carcinoma is included because EUS is used in its clinical staging.

It has been argued that patients with superficial tumors who undergo EMR and have specimens showing a positive deep margin or submucosal involvement should be referred for esophagectomy, ensuring a more thorough lymph node resection. EMR may thus have a diagnostic role for superficial esophageal cancers. ${ }^{11}$ Long-term follow-up data of EMR intramucosal and superficial submucosal lesions have shown a $61 \%$ overall 5 -year survival and no tumor-related deaths. ${ }^{10}$ These data are difficult to interpret, because many of those lesions had invasion only to the first one third of the submucosal layer (superficial submucosa), 
which in our institutional experience has been difficult to determine on EMR specimens. Also, the depth of mucosectomy associated with EMR varies. Our gastroenterologists use both the injection-snare and banding techniques when performing EMR. Regardless of the technique, our esophageal pathologists continue to see a varied depth of mucosectomy in EMR specimens. This may be due to the variation in volume of saline injected submucosally to raise the tissue and, subsequently, how much of the injection remains local to the desired resection site. In addition, in BE there is a duplicated lamina propria and muscularis mucosa, ${ }^{15}$ and more tissue superficial to the submucosa than in the normal squamous lined esophagus. This has been shown to affect the EUS staging accuracy of superficial EACs ${ }^{16}$ and may hinder the performance of EMR. One may be more likely to resect submucosa with normal squamous mucosa than when EMR is performed for BE. EMR may be a useful diagnostic tool but also has some limitations in use as definitive therapy.

RFA and PDT are other endoluminal therapies that are being used in an attempt to eradicate Tis and even some superficial EACs. However, unlike EMR, these modalities do not produce a tissue specimen for pathologic evaluation. Again, if EUS were used to qualify the use of RFA in the patients with early-stage esophageal cancer studied, 9 of the 90 patients thought to have $\mathrm{cN} 0$ would have persistent disease in involved lymph nodes. Also, tumor depth and its correlation with lymph node metastases would not be known because of the tissue ablative nature of the technology.

Despite the recent successes of EMR and RFA in abolishing luminal esophageal tissue, these therapies do not address nodal spread. Patients with Tis-T1 tumors have been shown to have a $24 \%$ rate of pathologic lymph node spread after esophagectomy, despite being staged by EUS to be N0. ${ }^{17}$ The cited study did not specify the depth of T1 tumor invasion or address the limitations of EUS for clinical decision-making, as our study did. It has also been reported that tumors confined to the mucosa have pathologically positive nodes $0 \%{ }^{18}$ to $6.9 \%$ of the time. ${ }^{19}$ This finding is consistent with our data of $9 \%(1 / 11)$ of pT1-mm tumor prevalence having pathologically positive lymph nodes. A number of studies have reported rates of $7.5 \%$ to $50 \%$ of patients with submucosal invasion and pathologically positive lymph nodes. ${ }^{13,20,21}$ However, in all of our T1a tumors (T1a-lp and Ta-mm), the positive lymph node prevalence was only $1.7 \%(1 / 59)$. This difference is a result of no lymph nodes being positive in T1a-lp tumors. Again, our data reveal a $17 \%$ rate $(6 / 36)$ of pathologically positive lymph nodes in pT1b-sm tumors, consistent with these studies. Thus, preoperatively predicting those patients with pT1a (pT1a-lp or pT1a-mm) tumors without resection is difficult.

Positron emission tomography (PET) is commonly used in the staging of esophageal malignancy. Our institution does not have a standard algorithm mandating the incorporation of PET in the staging of early-stage esophageal cancers, especially in tumors thought to be cTis. As a result, PET staging was not investigated in this study because of the lack of its uniform use in this subgroup of early-stage esophageal cancers.

The accuracy of EUS identifying metastatic disease in lymph nodes has been studied. Rampado and colleagues ${ }^{22}$ reviewed 55 patients with superficial carcinoma (T1a or T1b) and determined that NPV for EUS with nodal involvement was $84 \%$ and diagnostic accuracy of $71 \%$. These results are similar to the presented data that revealed nodal EUS NPV of $82.7 \%$ and accuracy of $75.7 \%$. Our study population did include a larger number of distal esophageal tumors $(90.7 \%$ vs $64 \%)$ and EACs $(83.1 \%$ vs $60 \%)$ than the referenced study. Others have reported the accuracy of EUS in detecting malignant adenopathy to vary from $50 \%$ to $70 \%{ }^{23,24}$ Also, the type of EUS probe can affect the accuracy, with mini-EUS probes being shown to be less accurate for determining $\mathrm{N}$-staging. ${ }^{22}$ The sensitivity and specificity of EUS for determining endoscopic therapy versus esophagectomy are poor for early-stage esophageal cancers. Others have demonstrated that standard EUS is not sufficient to distinguish mucosal from submucosal invading lesions. ${ }^{25,26}$ EUS routinely understaged lesions thought to be cT0-cT1a. Of the 46 tumors thought to be cT1aN0 on the basis of EUS, $5(11 \%)$ were shown to have at least $\mathrm{pN} 1$ disease. EUS is insufficient for determining lymph node involvement in early-stage esophageal cancers not treated with esophagectomy.

Fine-needle aspiration was performed in a minority of our patients $(\mathrm{n}=11)$, and all were found to be cytologically negative for inclusion in this study. Our endosonographers are not aggressive with regard to lymph node biopsy because of the likelihood that these patients will have a THE for their early-stage esophageal cancer. Thus, there may be some bias with regard to nodal EUS staging. Any patient with fine-needle aspiration-proven $\mathrm{pN} 1$ disease would have received neoadjuvant chemotherapy and radiotherapy preoperatively and was not included in this analysis.

Esophagectomy is a safe option in a high-volume center, particularly for early-stage esophageal cancers. The overall hospital mortality for this series was less than $1 \%$. This is in agreement with other high-volume centers where esophagectomy performed for Tis or intramucosal adenocarcinoma has been achieved with $0 \%$ mortality. ${ }^{9,19}$ The median interval of 59 days from the date of the EUS examination to the date of esophagectomy is attributable to our institutional protocol of preoperative pulmonary and physical rehabilitation before elective major surgery. The shortcoming of esophagectomy is the rate of overall perioperative complications, $33 \%$ in the presented series. In a recent study by Zehetner and colleagues, ${ }^{9}$ at another 
high-volume esophageal surgery center, the rate of overall esophagectomy morbidity was $39 \%$. More specifically, the perioperative morbidities associated with esophagectomy in our patients in this report, including anastomotic leak, recurrent laryngeal nerve injury manifested by transient hoarseness, wound infection, and chylothorax, were similar to our institution's previous report in 2007 on 2000 THEs. ${ }^{27}$ Despite a significant morbidity rate, our patients have had a high long-term satisfaction rate after esophagectomy ${ }^{28}$ a further endorsement for esophagectomy as the optimal choice for treatment even for early-stage cancers.

\section{CONCLUSIONS}

Endoluminal interventions, particularly EMR, will continue to play an important and likely increasing role in the diagnostic management of early-stage esophageal cancers. RFA, because of its inability to produce a diagnostic tissue specimen for pathologic review, has a limited role in early-stage esophageal cancer management. None of the current endoscopic-based therapies allow for evaluation of lymph nodes. EUS has shown to be inadequate for the definitive evaluation of lymph nodes in early-stage esophageal cancers, and in our experience has been poor at giving a reliable tumor depth assessment in this group. As a result, EUS should have a limited role beyond the initial staging examination to rule out more advanced lesions that would require neoadjuvant therapy before surgery. EMR may be a better staging tool for early-stage esophageal malignancies, whether used as an adjunct to EUS or in place of EUS. EMR is a promising staging tool, although it is not used routinely for staging at our institution. Esophagectomy is still the standard therapy for early-stage esophageal cancers in the majority of patients because it allows definitive staging of tumor depth and nodal involvement.

The authors thank Kay Perigo for management of the University of Michigan Esophagectomy Database.

\section{References}

1. Siegel R, Naishadham D, Jemal A. Cancer statistics, 2013. CA Cancer J Clin. 2013;63:11-30

2. Leers JM, DeMeester SR, Oezcelik A, Klipfel N, Ayazi S, Abate E, et al, The prevalence of lymph node metastases in patients with T1 esophageal adenocarcinoma a retrospective review of esophagectomy specimens. Ann Surg. 2011;253:271-8.

3. Enzinger PC, Mayer RJ. Esophageal cancer. N Engl J Med. 2003;349: 2241-52.

4. Zehetner J, DeMeester SR. Treatment of Barrett's esophagus with high-grade dysplasia and intramucosal adenocarcinoma. Expert Rev Gastroenterol Hepatol. 2009:3:493-8.

5. Dye CE, Waxman I. Endoscopic ultrasound. Gastroenterol Clin North Am. 2002; 31:863-79.

6. Twine CP, Lewis WG, Escofet X, Bosanquet D, Ashley Roberts S. Prospective comparison of optic versus blind endoscopic ultrasound in staging esophageal cancer. Surg Endosc. 2009;23:2778-84.

7. Sobin LH, Gospodarowicz MK, Wittekind C. TNM Classification of Malignant Tumors. 7th ed. New York, NY: Wiley-Blackwell; 2010.
8. Pech O, Bollschweiler E, Manner H, Leers J, Ell C, Holscher AH. Comparison between endoscopic and surgical resection of mucosal esophageal adenocarcinoma in Barrett's esophagus at two high-volume centers. Ann Surg. 2011;254:67-72.

9. Zehetner J, DeMeester SR, Hagen JA, Ayazi S, Augustin F, Lipham JC, et al. Endoscopic resection and ablation versus esophagectomy for high-grade dysplasia and intramucosal adenocarcinoma. J Thorac Cardiovasc Surg. 2011; 141:39-47.

10. Prasad GA, Wang KK, Buttar NS, Wongkeesong LM, Krishnadath KK Nichols FC III, et al. Long-term survival following endoscopic and surgical treatment of high-grade dysplasia in Barrett's esophagus. Gastroenterology. 2007; 132:1226-33.

11. Maish MS, DeMeester SR. Endoscopic mucosal resection as a staging technique to determine the depth of invasion of esophageal adenocarcinoma. Ann Thorac Surg. 2004;78:1777-82.

12. Buskens CJ, Westerterp M, Lagarde SM, Bergman JJ, ten Kate FJ, van Lanschot JJ. Prediction of appropriateness of local endoscopic treatment for high-grade dysplasia and early adenocarcinoma by EUS and histopathologic features. Gastrointest Endosc. 2004;60:703-10.

13. Raja S, Rice TW, Goldblum JR, Rybicki LA, Murthy SC, Mason DP, et al Esophageal submucosa: the watershed for esophageal cancer. J Thorac Cardiovasc Surg. 2011;142:1403-11.e1.

14. Manner H, May A, Pech O, Gossner L, Rabenstein T, Gunter E, et al Early Barrett's carcinoma with "low-risk" submucosal invasion: long-term results of endoscopic resection with a curative intent. Am J Gastroenterol. 2008;103:2589-97.

15. Lewis JT, Wang KK, Abraham SC. Muscularis mucosae duplication and the musculo-fibrous anomaly in endoscopic mucosal resections for Barrett esophagus: implications for staging of adenocarcinoma. Am J Surg Pathol. 2008;32: 566-71.

16. Mandal RV, Forcione DG, Brugge WR, Nishioka NS, Mino-Kenudson M, Lauwers GY. Effect of tumor characteristics and duplication of the muscularis mucosae on the endoscopic staging of superficial Barrett esophagus-related neoplasia. Am J Surg Pathol. 2009;33:620-5.

17. Crabtree TD, Yacoub WN, Puri V, Azar R, Zoole JB, Patterson GA, et al Endoscopic ultrasound for early stage esophageal adenocarcinoma: implications for staging and survival. Ann Thorac Surg. 2011;91:1509-16.

18. Stein HJ, Feith M, Mueller J, Werner M, Siewert JR. Limited resection for early adenocarcinoma in Barrett's esophagus. Ann Surg. 2000;232:733-42.

19. Pennathur A, Farkas A, Krasinskas AM, Ferson PF, Gooding WE Gibson MK, et al. Esophagectomy for T1 esophageal cancer: outcomes in 100 patients and implications for endoscopic therapy. Ann Thorac Surg. 2009;87:1048-55.

20. Sepesi B, Watson TJ, Zhou D, Polomsky M, Litle VR, Jones CE, et al Are endoscopic therapies appropriate for superficial submucosal esophageal adenocarcinoma? An analysis of esophagectomy specimens. J Am Coll Surg. 2010;210:418-27.

21. Siegel R, Naishadham D, Jemal A. Cancer statistics, 2012. CA Cancer J Clin 2012;62:10-29.

22. Rampado S, Bocus P, Battaglia G, Ruol A, Portale G, Ancona E. Endoscopic ultrasound: accuracy in staging superficial carcinomas of the esophagus. Ann Thorac Surg. 2008;85:251-6.

23. Rice TW, Zuccaro G Jr, Adelstein DJ, Rybicki LA, Blackstone EH, Goldblum JR Esophageal carcinoma: depth of tumor invasion is predictive of regional lymph node status. Ann Thorac Surg. 1998;65:787-92.

24. Rice TW, Blackstone EH, Adelstein DJ, Zuccaro G Jr, Vargo JJ, Goldblum JR, et al. Role of clinically determined depth of tumor invasion in the treatment of esophageal carcinoma. J Thorac Cardiovasc Surg. 2003;125:1091-102.

25. Pech O, May A, Gunter E, Gossner L, Ell C. The impact of endoscopic ultrasound and computed tomography on the TNM staging of early cancer in Barrett's esophagus. Am J Gastroenterol. 2006;101:2223-9.

26. Pouw RE, Heldoorn N, Herrero LA, ten Kate FJ, Visser M, Busch OR, et al Do we still need EUS in the workup of patients with early esophageal neoplasia? A retrospective analysis of 131 cases. Gastrointest Endosc. 2011; 73:662-8.

27. Orringer MB, Marshall B, Chang AC, Lee J, Pickens A, Lau CL. Two thousand transhiatal esophagectomies: changing trends, lessons learned. Ann Surg. 2007; 246:363-74.

28. Davis SJ, Zhao L, Chang AC, Orringer MB. Refractory cervical esophagogastric anastomotic strictures: management and outcomes. J Thorac Cardiovasc Surg. $2011 ; 141: 444-8$. 


\section{Discussion}

Dr Steven DeMeester (Los Angeles, Calif). The authors reviewed their database from July 2005 to 2011 for clinically staged patients, carcinoma in situ or T1, and had biopsies showing high-grade dysplasia or invasive cancer. All the patients had EUS, and those with EUS, clinical stage T2 or greater, biopsy-proven N1 disease, and neoadjuvant therapy were excluded. The final resection pathology was then used to assess the accuracy of EUS for $\mathrm{T}$ and $\mathrm{N}$ staging.

My first question is why did the authors choose to go at it seemingly backward? In other words, why not start with all pathologically staged high-grade dysplasia or T1 cancers and go back and analyze how these patients were clinically staged? Given the shown and known inaccuracy of EUS for both T and N staging, how many of the incorrectly staged T patients, such as those staged T2 clinically and were therefore excluded from your analysis, might have actually been T1a lesions that you should have included, and, likewise, by excluding known N1 disease, how many of those patients might have had a submucosal tumor and were therefore excluded, thereby altering the true prevalence of your nodal disease in this patient group?

Dr Bergeron. We chose to select first for the clinical staging based on EUS because this is the typical decision-making process that occurs for patients who are to undergo endoluminal therapies. That is what led to our study design.

Dr DeMeester. It might be worth looking backward at it again and just see if you come up with different numbers because that is an important issue with the mis-staging that you have demonstrated.

It has been well demonstrated that even with high-frequency 20 $\mathrm{MHz}$ ultrasound probes, differentiation between lamina propria and muscularis mucosa invasion is difficult. I would suggest that it is simply not possible with standard 7.5 and $12 \mathrm{MHz}$ probes. What was the impetus to try to distinguish between those with 12.5 or $7.5 \mathrm{MHz}$ probes, the standard probes? Who was doing the re-reviews? Was this you or your gastroenterology colleagues or a combination of them?

Dr Bergeron. It was a combination as far as the review. The 20 $\mathrm{MHz}$ probes, some claim to be able to differentiate lamina propria from muscularis mucosa invasion. However, surprisingly, in the majority of the EUS reports, which included only the 7.5 or $12 \mathrm{MHz}$ probes, the gastroenterologists performing the EUS did differentiate that the tumor was invading the lamina propria versus the muscularis mucosa. This information was routinely included.

Dr DeMeester. I would suggest that as someone who does them himself, that's simply not possible. Did you re-review the final pathology as well? Oftentimes pathologists, particularly a number of years ago before endoscopic resection became an important part of the staging system, weren't differentiating between T1a and T1b because at that time it was all T1 lesions for them. Did you go back and look at the pathology or had they already done a good job of determining the precise depth of invasion on your final pathology?

Dr Bergeron. In all of our pathologic analysis, T1a was differentiated from T1b in the original final pathology reports.

Dr DeMeester. You demonstrated that there was only 1 patient with an intramucosal tumor who had lymph node positivity. He had invasion into the muscularis mucosa layer. But you had a small number of patients, only 11, with invasion into the muscularis mucosa. Larger series, such as ours, in which we had in excess of 50 patients with invasion to the muscularis mucosa level demonstrated only 1 patient. In other words, if you just look at the mucosal layer alone, only $1.7 \%$ of your patients had a positive lymph node, which compares favorably to other series. Are you factoring that in, or are you choosing to select muscularis mucosal invasion as a marker against endoscopic therapy, sort of in contrast to what is accepted by most other centers for endoscopic therapy?

Dr Bergeron. Yes, that is correct. In our experience, we had only 1 tumor that invaded the muscularis mucosa that did have positive nodes, and if you look at a lot of the endoscopic or endoluminal-based therapy studies, there is a low percentage rate of positive nodes with mucosal invasion. However, in a previously reported study looking at minimally invasive esophagectomy specimens, they had an approximate $6 \%$ rate of positive lymph nodes with invasion into the muscularis mucosa. The take-away from our review is that simply not just mucosal invasion would be an indication for endoluminal therapy, but potentially the more superficial lesion with only involvement of the lamina propria might be more acceptable.

Dr DeMeester. Just one quick comment. I know your final slide said that esophagectomy should still be considered the standard of care. I suggest that unless we as surgeons embrace endoscopic therapy and take it on and incorporate it into our practices, I fear for our specialty's long-term involvement in the esophagus.

Dr Robert Cerfolio (Birmingham, Ala). Our experience has been similar, and I want to echo Steve's point about us needing to be involved. I have 2 quick questions. One, did you stratify your data based on the endosonographer, that is, you versus the gastroenterologist?

Dr Bergeron. All of the endosonography that was performed was performed by gastroenterologists in this study.

Dr Cerfolio. I thought you said you were doing it when Steve asked you.

Dr Bergeron. No. We reviewed the EUS studies, but thoracic surgeons were not the physicians performing the endosonography.

Dr Cerfolio. So you mean you were just looking at the ultrasound after they would do it?

Dr Bergeron. Yes. Or we were able to take the level of sublevel of lesion penetration depth directly from the report as described by the endosonographer.

Dr Cerfolio. That's not the way to do it. I mean if you're going to do it, be the endosonographer and get involved. It would be interesting to see if surgeons do it differently. Certainly with endobronchial ultrasound, I don't think there are data yet, but I think it's coming that we're going to be a little bit better at it, and I wonder if that applies to EUS. I would like to see data on that.

You didn't show me what you did with the patient who has T1N1, unsuspected N1. Are you giving him adjuvant chemotherapy when you present him at their tumor conference? We have had incredibly heated debates. I know I don't know what to do. Maybe you can tell me what to tell this patient who I have to call later today about unsuspected N1 disease; 41 nodes were taken out, and he had $2 \mathrm{~N} 1$ nodes positive. He did not get 
neoadjuvant therapy. Do you treat him, and if you treat him, what do you treat him with? He's a healthy guy, can tolerate anything, doing great, and Eastern Cooperative Oncology Group status is zero.

Dr Bergeron. I don't know the answer to that. In general, I would suspect that a patient with nodal disease would receive adjuvant therapy at our institution if age were not prohibitive.
Dr Cerfolio. You don't know if they were treated?

Dr Bergeron. I don't.

Dr Cerfolio. Anybody in the audience want to educate me as to what they're doing? (Inaudible voices from the audience.)

Dr Cerfolio. Mark Krasna said postoperative radiation and chemotherapy. Dr DeMeester said less than 3 nodes, don't do anything. Interesting. 